\title{
Determination of Simultaneous Steady-State Movements Using Blade Tip Timing Data
}

DOI:

$10.1115 / 1.4045265$

\section{Document Version}

Accepted author manuscript

Link to publication record in Manchester Research Explorer

\section{Citation for published version (APA):}

Mohamed, M., Bonello, P., \& Russhard, P. (2019). Determination of Simultaneous Steady-State Movements Using Blade Tip Timing Data. Journal of Vibration and Acoustics. https://doi.org/10.1115/1.4045265

\section{Published in:}

Journal of Vibration and Acoustics

\section{Citing this paper}

Please note that where the full-text provided on Manchester Research Explorer is the Author Accepted Manuscript or Proof version this may differ from the final Published version. If citing, it is advised that you check and use the publisher's definitive version.

\section{General rights}

Copyright and moral rights for the publications made accessible in the Research Explorer are retained by the authors and/or other copyright owners and it is a condition of accessing publications that users recognise and abide by the legal requirements associated with these rights.

\section{Takedown policy}

If you believe that this document breaches copyright please refer to the University of Manchester's Takedown Procedures [http://man.ac.uk/04Y6Bo] or contact uml.scholarlycommunications@manchester.ac.uk providing relevant details, so we can investigate your claim.

\section{OPEN ACCESS}




\title{
DETERMINATION OF SIMULTANEOUS STEADY-STATE MOVEMENTS USING BLADE TIP TIMING DATA
}

\author{
Mohamed Elsayed Mohamed \\ Department of Mechanical, Aerospace and Civil Engineering, University of Manchester, M13 9PL, \\ Manchester, United Kingdom \\ Faculty of Engineering, Cairo University, Cairo, Egypt \\ mohamed.mohamed-2@manchester.ac.uk \\ Philip Bonello \\ Department of Mechanical, Aerospace and Civil Engineering, University of Manchester, M13 9PL, \\ Manchester, United Kingdom \\ Philip.Bonello@manchester.ac.uk
}

\section{Peter Russhard}

EMTD Ltd, NG8 2RD, Nottingham, United Kingdom

peter.russhard@emtd-measurement.com

\begin{abstract}
Blade tip timing (BTT) includes a number of uncertainties that discourage its use. One of the main ones is the shift in the equilibrium position of the blade tip due to steady (non-oscillatory) bending and/or twisting of the blade, and axial movement of the bladed disk (blisk)-shaft system. This results in a shift in the effective measurement position of the probe relative to the blade chord, resulting in errors in the tip vibration measurement which can translate to a huge error in the corresponding stress estimate, which relies on calibration against finite element (FE) models. Previous experimentally validated research by the authors introduced a method for quantifying steady movement of a single type (axial, lean, or untwist), using BTT data from not more than two probes. In this paper, a development of the previous method is presented that provides a solution for the case of simultaneous types of blade steady movements. Additional probes are used for determining the direction, but these can be placed at any angular positions. The developed method is validated using a BTT simulator of a blisk, and accurate results obtained. The simultaneous axial and lean movements can be accurately determined when the untwist is negligible, and an uncertainty level can be specified when the untwist is not negligible.

The untwist itself can be calculated accurately in all cases of simultaneous movements. Guidelines for the use of the method in different scenarios are provided.
\end{abstract}




\section{NOMENCLATURE}

\begin{tabular}{|c|c|}
\hline Blisk & Bladed disk \\
\hline BTT & Blade tip timing \\
\hline CFF & Circumferential Fourier Fit \\
\hline DOF & Degree of freedom \\
\hline EO & Engine Order \\
\hline FE & Finite element \\
\hline NSMS & Non-Intrusive Stress Measurement System \\
\hline OPR & Once per revolution probe \\
\hline SGs & Strain gauges \\
\hline$d_{j}$ & Blade tip displacement at probe $j$ \\
\hline $\mathrm{EO}_{m}$ & Engine order, $m=1, \ldots M$ \\
\hline$j$ & Probe number \\
\hline$l$ & Distance between probe and intersection point \\
\hline$M$ & Number of excitation frequencies \\
\hline$n$ & Revolution number \\
\hline$N_{\text {buff }}$ & Number of buffers for averaging \\
\hline$N_{\mathrm{P}}$ & Number of probes \\
\hline$\left.N_{P}\right|_{\text {min }}$ & Minimum number of probes \\
\hline$o$ & Origin point \\
\hline$P_{j}$ & The mean displacement value at probe $j$ \\
\hline$R$ & Blade tip radius \\
\hline$S$ & Magnitude of combined axial-lean movement \\
\hline$t$ & Time \\
\hline$t_{\mathrm{det}}$ & Detected arrival time of blade tip \\
\hline$t_{\exp }$ & Expected arrival time of blade tip \\
\hline
\end{tabular}




$\begin{array}{ll}x, y, z & \text { Cartesian coordinates } \\ X_{r} & \text { Carrington's cross correlation factor } \\ z_{\text {min }}, z_{\max } & \text { Axial positions of blade tip edges } \\ \alpha_{j} & \text { Probe angle relative to OPR } \\ \beta_{i} & \text { Blade angle relative to OPR } \\ \Delta P_{j} & \text { Change in probe offset } \\ \Delta P_{u} & \text { Change in probe offset due to only untwist } \\ \Delta z_{\mathrm{c}} & \text { Distance between probe and twisting centre } \\ \Delta z_{\mathrm{p}} & \text { Axial distance between probes } \\ \emptyset & \text { Blade chord line angle } \\ \emptyset_{S} & \text { Axial-Lean movement angle } \\ \psi & \text { Untwist angle } \\ \omega_{m} & \text { Vibration frequency } m=1, \ldots . M \\ \Omega_{m} & M\end{array}$

\section{INTRODUCTION}

Excessive blade vibration results in blade failure during operation. Measurement of blade vibration (amplitude and frequency) makes it possible to avoid such failures and protect different components [1]. It also provides the designers of bladed assembly components in turbomachinery applications with the information that is necessary for determining the limits posed by the harsh operating conditions (high speed, temperature, and pressure), and to improve the design accordingly [2]. Two types of blade vibration can occur: 1) synchronous (Integral EO) which results from non-uniform pressure distributions due to stators and/or irregularities in the casing, 2) asynchronous (non-integral EO), which results from rotating stall and/or aerodynamic instabilities [3].

Strain gauges (SGs) are the most commonly used devices for monitoring blade vibration, providing direct information about the blades stresses [4]. However, their attachment to the blades may affect the blade dynamics. Also, the measurements are limited to a few blades and require considerable instrumentation since the signals have to be transmitted via telemetry or a slip ring [3]. The SG service life is short, making it unsuitable for long-term measurements [5]. Another blade vibration measurement method which has limitations similar to SGs is the frequency modulated grid system, which uses small permanent magnets attached to the blades tips [6]. 
In contrast to these methods, BTT provides an advanced blade vibration technique while fulfilling the requirements of a low cost and effective vibration measurement system [7], [3].

The principle of BTT systems is to use a number of probes (generally optical probes) mounted on the casing, with laser light beams focusing onto the passing blades tips. When a blade tip intersects with the light beam, the light is reflected back to a photo sensor and light intensity rises at a high rate. The time at which a blade tip passes within the range of a probe is called arrival time. In case of no vibration and/or steady blade movements, the arrival time would be only dependant on the rotational speed and angular positions of both the blade and the probe (this is called the "expected arrival time"). However, in normal cases, the blade arrival times are dependent on the vibration (amplitude and frequency), and the steady (non-oscillatory) movement (its type and rate), experienced by the blade [7]. Consequently, the blades will pass the probes earlier or later than the expected time, and the difference between the actual and expected arrival times of a blade at a given probe is a measure of the instantaneous tip displacement over and above the rigid body rotational motion. Such displacements ("BTT data") are processed by a BTT algorithm for frequency, amplitude and phase information. Since BTT measurements refer to the blade tip, their conversion to stress information at critical points on the blade requires prior calibration against strain gauge (SG) measurements or finite element (FE) simulations.

Pioneering work on BTT started 50 years ago [3]. It was first known as Non-Intrusive Stress Measurement System (NSMS) [8-10], then it has been subjected to many developments with regard to both the instrumentation/data acquisition aspect, and the BTT data analysis algorithms aspect. During the last two decades, a considerable development for the data analysis algorithms of the current BTT technology has been done started by the work of Heath [11-13], and then Dimitriadis et al. developed the Auto-regressive methods [14-16]. Another method which is considered as a standard method used in a leading aero-engine company has been presented by Russhard [17], it is known as multi-frequency sine fitting with preparation method. It involves the preparation of data to separate integral from non-integral vibration components. Other attempts include the recent improvement has been done by Rigosi et al. [18] to the early two-parameter plot method introduced by Heath [12], in addition to the work of Jun Lin et al. [19] for monitoring multi-mode vibration signals. Moreover, different techniques have been produced inside industry and kept confidential, or have been published with little data, like the circumferential Fourier Fit (CFF) method [20].

The correlation process between the measured BTT displacements and FE predictions involves a number of uncertainties relating to measurement, data processing/analysis, and FE modelling [21,22]. The change in the equilibrium position of the blade tip as a result of steady (non-oscillatory) movement of the blades is one of the major causes of uncertainty in BTT measurements. With reference to Figure 1, the three principal types of steady movements of the blade tip are: 
- Axial shift, where the direction of movement of the blade tip chord line is perpendicular to the plane of rotation (Figure 1a);

- Blade lean, where the blade tip is moving perpendicular to the blade chord line (Figure 1b),

- Blade untwist, where the blade is twisting around an axis parallel to the longitudinal axis of the blade as shown in Figure 1c.

Such steady movement is typically caused by variations in thermal and centrifugal loading conditions that are associated with changes in the rotational speed.

The BTT measurement uncertainty introduced by such steady movement is the result of the shift in the effective measurement (sensing) positions of the BTT probes relative to the blade tip. This deviation from the nominal measurement position manifests itself as a "DC" error in the BTT data which affects the accuracy of the amplitude and frequency output by the BTT algorithm, since the amplitudes of vibration at the original and effective measurement positions may be significantly different. This, in turn, can have a significant impact on the stress estimate (based on the pre-determined calibration factor linking the stress values of the blisk to the tip deflection). Moreover, the new measurement point might have minimal amplitude of vibration of a certain mode shape which results in that mode being missed. Sometimes, the blade may be completely missed from measurements due to position shift if the probe placed close to the edges [23].

It is important to note that the effect of steady movements is neglected in the current BTT data algorithms, while some of them consider only the effect of angular position offsets of the probes which are independent of the rotational speed. As for the determination of the steady movement itself, the methods that have been proposed typically require additional equipment or are restricted to certain types of blades [24-28]. A few attempts have been published about the identification and quantification of blade steady movements and their effect on BTT measurements. Some of these attempts require the use of additional probes and may be predictions of the mode shapes to determine the probe sensing positions due to steady movements such as the one described in [24], and the method presented by Twerdochlip et al. [25] for the determination of blade untwist under synchronous dynamic conditions. This method depends on the measurement of blade passing signals using two pairs of fixed sensors. The signals from both pairs are then evaluated to distinguish between blade untwist and synchronous vibration. Other methods use additional equipment such as the one presented by Hatcher Jr et al. [26] which uses an image capture device for capturing the blade tip simultaneously with the detected arrival time, with the sensing position being located either manually or using image processing. Kominsky [27] introduced a method for the calculation of the blade untwist angle by measuring the intensity of light reflected from the blade using optical fibres. The 
method proposed by Olivier Jousselin [28] extracted the shifted measurement position in case of lean, axial shift, or untwist, but was limited to shrouded turbine blades since the method depended on special geometric features associated with such components. Moreover, neither experimental nor simulated data validation was presented for the method of [28].

A novel method has been presented by the authors [23] for the determination of individual blade steady movements (axial shift, blade lean, and blade untwist) using BTT data. This method depends on the extraction of the change in the average values of BTT displacements measured by only one or two probes, depending on the type of movement, and then linking it to the blade movement by means of geometrical relations. The work in [23] included preliminary validation using a novel BTT simulator of a blisk. Experimental validation tests using measurements from both a laboratory test rig and real engines were then undertaken and their results are presented in [29]. Despite the proven validity of the method in [23,29], it was devised for each individual type of movement occurring separately (as in Figures 1a-c). However, in most real engine measurements it is usual to find these movements occurring simultaneously. Thus, a development of the authors' previous method $[23,29]$ is essential in order for it to be applicable to all movement situations, and to quantify both the magnitude and direction of such movements accurately.

This paper therefore presents a new development of the above mentioned method [23,29] that is aimed towards the determination of steady movements that are a combination of more than one type of the basic steady movements, as illustrated in Figure 1d. The new methods are validated using the blisk modal model simulator [23]. It should be noted that the developed method is valid for any bladed disk, not only disks with integrated blades as the one used in this work. The following sections include an explanation of BTT systems, the problem of BTT measurement position errors, and a description of the developed method for the determination of simultaneous steady movements. A brief description of the simulator and the blisk FE model used in the validation process is then presented. This is then followed by a discussion of the results of the method, and the conclusions of the work.

\section{EFFECT OF STEADY MOVEMENTS ON BTT MEASUREMENTS}

In order to understand the influence of steady movements on BTT displacement measurements, consider the blisk shown in Figure 2 which is rotating about the $z$-axis. The expected arrival time $t_{\text {exp }}$ of the tip of the shown blade (blade no. $i$ ) at probe no. $j$ is dependent on the rotational speed $\Omega$, which is assumed constant during every cycle of rotation, and can be calculated from the OPR signal probe as follows

$t_{\mathrm{exp}, j}=t_{\mathrm{OPR}, n}+\left(\alpha_{j}-\beta_{i}\right) / \Omega$ 
where $t_{\mathrm{OPR}, n}$ is the start time of revolution no. $n, \alpha_{j}$ is the angular position (in $x-y$ plane) of the probe $j$ relative to the OPR probe, and $\beta_{i}$ is the angular position of the nominal sensing point on the tip of the undeformed blade relative to the OPR reference feature on the shaft (see Figure 2). The actual (detected) arrival time $t_{\text {det }}$ would be either greater, or less than the expected time depending on the vibration behaviour of the blade. The tip displacement of the blade as it passes probe $j$ is then estimated as follows [30]

$d_{j}=\Omega *\left(t_{\mathrm{exp}}-t_{\mathrm{det}}\right) * R$

where $R$ is the tip radius measured from the centre of rotation.

The instantaneous tip displacements $d_{j}$ of a given blade observed by probe no. $j$ determined from the above formula are assumed to follow the following variation in the time domain [29]:

$$
\begin{gathered}
d_{j}=P_{j}+\sum_{m=1}^{M}\left(a_{1 m} \sin \left(\mathrm{EO}_{m} \Omega t_{j}\right)+a_{2 m} \cos \left(\mathrm{EO}_{m} \Omega t_{j}\right)\right) \\
+ \text { noise }
\end{gathered}
$$

where, $\omega_{m}=\mathrm{EO}_{m} \Omega, m=1 \ldots M$, is a generic frequency component, and $\mathrm{EO}_{m}$ is the associated engine order (EO). The harmonic components can be either synchronous $\left(\mathrm{EO}_{m}\right.$ integer) or non-synchronous $\left(\mathrm{EO}_{m}\right.$ non-integer). $t_{j}$ in eq. (3) represents the sampling time according to the assumptions made in the BTT algorithm used. $P_{j}$ is the steady offset resulting from the positional (fixing) errors of the probes and/or blades, in addition to the blade steady movements. Positional errors and blade steady movements introduce errors in $t_{\text {exp, } j}$ (eq. (1)), and therefore $d_{j}$, which cause $P_{j}$. Positional error in the probes affects $\alpha_{j}$, whereas positional (fixing) error in the blade affects $\beta_{i}$ (see Figure 2). Such angular errors are speed-independent, and, due to division by $\Omega$ in eq. (1) and multiplication by $\Omega$ in eq. (2), the resulting error in $d_{j}$ is also independent of speed $\Omega$. Hence, the contribution to the mean term $P_{j}$ arising purely from probe or blade positional offset is independent of speed, as seen in Figure 3a. On the other hand, the shift in sensing position introduced by blade steady movements results in a speed-dependent error in $\beta_{i}$ (Figure 2) which translates into a speed-dependent mean term $P_{j}$, as shown in Figure $3 \mathrm{~b}$. 
Starting from this observation, the change in average values of BTT displacements at a probe $j$ between two different speed values can be used to quantify the type and amount of steady movement of the blade tip by using the geometrical relations as described in the following section.

\section{BLADE STEADY MOVEMENTS DETERMINATION}

Based on previous work for the determination of individual type movements (either axial or lean or untwist) [23,29] it is only possible to distinguish between untwist and either axial or lean by comparing $\Delta P_{j}$ of two different probes as follows

If $\Delta P_{1}=\Delta P_{2} \quad$ Axial or lean

If $\Delta P_{1} \neq \Delta P_{2} \quad$ untwist

where $\Delta P_{1}$ and $\Delta P_{2}$ are the changes in average values between two different rotational speeds at two different probes. The two probes have to be placed at different axial positions $\left(z_{1} \neq z_{2}\right)$, but they can be placed at either the same or different angular positions $\left(\theta_{1}, \theta_{2}\right)$. The geometrical relations for individual types of movements are available in [23,29]. The following sections present a development of this previous method to cater for all movement situations. It should be noted that the geometrical relations extracted in the following sections are based on the chord line of the blade tip regardless of its profile, which means that the current method can be used for any blade tip profile.

\subsection{Extraction of BTT displacement averages}

As in $[23,29]$, the first step is the extraction of the speed-varying offset $P_{j}$ as the speed is swept over a large number of revolutions. The method is based on that introduced by Russhard [17] for BTT data preparation. The BTT displacement data $d_{j}$ are divided into a large number of segments (buffers). Each buffer consists of a number of points $N_{\text {buff, }}$, where every point represents a revolution. The offset associated with the data in each buffer is then computed and this will be the offset $P_{j}$ associated with the average rotational speed for the buffer. $P_{j}$ for a given buffer is determined by calculating the simple arithmetic average of the data in the buffer, except when the buffer lies within the resonance region. In case of BTT data with integral EO vibration (eq. (3)), the change in BTT displacement values $d_{j}$ from a revolution to the next against the gradual variation of rotational speed is small (no change for constant speed). Thus, a large amount of vibration displacement would be included in the extracted offset $P_{j}$, which is acceptable outside the resonance regions that have low amplitude of vibration. However, within the region of resonance the buffer averages would change 
rapidly due to the change in vibration amplitude, and then it would conflict with the gradual change of $P_{j}$ resulting from steady movements. Hence, a mask is placed on the resonance region and its average values are calculated by linear interpolation between those of the buffers just before and after the resonance segment.

In order to identify the resonance regions to be masked, the coherence of the displacement data from a number of probes, based on a revolution-by-revolution technique, is examined using the cross correlation factor introduced by Carrington [31].

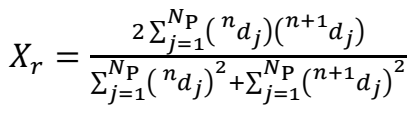

where ${ }^{n} d_{j}$ is the displacement of a given blade observed by probe $j$ during revolution $n$. According to [17], the value of $X_{r}^{2}$ should be close to 0.99 based on experiments for amplitudes in the resonance region of at least $0.1 \mathrm{~mm}$. Also, it was reported that the minimum number of probes required for $X_{r}$ to be used depends on the number of excitation frequencies $M$, and can be calculated as

$\left.N_{P}\right|_{\min }=2 M+2$

Note that if the number of probes is less than the $\left.N_{P}\right|_{\min }, X_{r}$ would always give a value of 1 [17]. Now the change in offset values $\Delta P_{j}$ can be extracted and employed in different geometrical relations to quantify the steady movements of the blades tips. Also, the conditions in eq. (4) can be modified for generic movement so that they indicate if there is untwist movement or not

If $\Delta P_{1}=\Delta P_{2} \quad$ No untwist (Axial and/or lean)

If $\Delta P_{1} \neq \Delta P_{2} \quad$ Untwist (other movements may exist)

\subsection{Axial-Lean}

Figure 4 shows a blade tip subjected to simultaneous axial and lean movements, which result in a combined movement of a general direction with unknown angle, in addition to the unknown amount of movement. The original equilibrium position of the blade tip is represented by the solid line, while the dashed line represents the displaced blade tip. Both lines have the same angle $\emptyset$ with respect to the horizontal line parallel to the $z$-direction (the rotational axis). $z_{1}, z_{2}, z_{\min }$ and $z_{\max }$ are the respective axial positions of probes 1 , 2, and the right and left edges of the blade tip according to the view shown in the figure. Points $a$ and $b$ are the measurement positions of probes 1 and 2 along the blade tip before the movement, while $a^{\prime}$ and $b^{\prime}$ are the new positions of these two points after the movement. Hence, $\overline{a a^{\prime}}$ and $\overline{b b^{\prime}}$ are equal to the magnitude of movement $S$ and have the same direction $\emptyset_{S}$. Meanwhile, probes 1 and 2 
have two different measurement positions $\left(a^{\prime \prime}\right.$ and $\left.b^{\prime \prime}\right)$ along the blade tip after movement, since the original points moved to different axial positions. The changes in probes offsets in the direction of rotation $(\Omega R)$ are equal to $\overline{a a^{\prime \prime}}$ and $\overline{b b^{\prime \prime}}$ which have the same value $\Delta P$.

Considering the triangle $\left(a-a^{\prime}-a^{\prime \prime}\right)$ shown in the top left side of Figure 4, the magnitude of movement can be calculated as

$$
S=\frac{\Delta P \cos \emptyset}{\sin \left(\emptyset+\emptyset_{S}\right)}
$$

The calculation of $S$ using the last formula depends on the value of $\emptyset_{S}$, which is also required to identify the direction of movement. This means that more information is required, which is provided by additional two probes ( 3 and 4 ) added at axial positions $z_{3}$ and $z_{4}$ according to the following conditions

$$
\begin{aligned}
& z_{3}<z_{\text {min }} \\
& z_{4}>z_{\text {max }}
\end{aligned}
$$

Consider now the triangle $\left(c-c^{\prime}-d\right)$ shown in the top right side of Figure 4, in which points $c$ and $c^{\prime}$ represent the right edge of the blade tip before and after the movement, and point $d$ is the intersection point between the extension of the solid line and a vertical line passing by point $c^{\prime}$. The following formula for $\emptyset_{S}$ can be deduced, noting that $\left(c^{\prime} d\right)$ is equal to $\Delta P$ detected by either probe 1 or 2 :

$$
\emptyset_{S}=\tan ^{-1}\left(\frac{\Delta P}{\left|z_{\min }-z_{3}\right|}-\tan \emptyset\right)
$$

In order for the above formula in eq. (10) to be valid, the measurements of probe 3 should be monitored over the range of rotational speeds to ensure that the steady movement has reached a sufficient magnitude for the blade to be detected by probe (3).

According to the direction of movement shown in Figure $4, \emptyset_{S}$ would have a value between zero (only axial movement) and $90-\emptyset$ (only lean movement). It should be noted that $\emptyset_{S}$ is assumed to be constant over all the measurements. Knowing the direction of movement $\left(\emptyset_{S}\right)$, its magnitude $S$ can be calculated using eq. (8). Note that, eq. (10) can also be used when the blade tip moves towards probe 4 , however the term $z_{\min }-z_{3}$ should be replaced by $z_{\max }-z_{4}$. Also, it should be noted that $\emptyset_{S}$ is assumed constant all over the measurements.

\subsection{Axial-Lean-Untwist}


The blade tip shown in Figure 5 is subjected to combined axial-lean $(S)$, and untwist $(\psi)$ movements simultaneously. The solid and dashed lines represent the original and moved equilibrium positions of the tip respectively. Points $a, b$ and $a^{\prime}, b^{\prime}$ represent the measurement positions of probes 1 and 2 before and after movement respectively, point $o$ is the intersection point of the blade lines before and after movement (it will be the twisting centre point in case of no axial-lean movement), and $\Delta z_{p}$ is the axial distance between the two probes. As made clear in section 3.2, the axial-lean type of movement does not change the chord line angle. This means that the untwist angle $\psi$ is not affected by other types of movement, and it can be calculated using $\Delta P$ values from two probes placed at different axial positions and the initial chord line stagger angle $\emptyset$, based on the triangles $\left(o-a-a^{\prime}\right)$ and $\left(0-b-b^{\prime}\right)$ shown in Figure 5 as follows

$$
\psi=\varnothing-\tan ^{-1}\left(\frac{l \sin \emptyset-\Delta P_{1}}{l \cos \emptyset}\right)
$$

and

$$
l=\frac{\Delta P_{1} \Delta z_{p}}{\left(\Delta P_{1}-\Delta P_{2}\right) \cos \varnothing}
$$

In Figure 5: $\overline{a a^{\prime}}$ and $\overline{b b^{\prime}}$ are equal to the offset values $\Delta P_{1}$ and $\Delta P_{2}$ of probes 1 and 2 respectively, which are different as per condition in eq. (7b); $l$ is the distance between points $o$ and $a^{\prime}$.

In contrast to the untwist, axial-lean movement cannot be determined using the detected values of $\Delta P$ until the untwist shift is reduced or subtracted from the measured data. This can only be done if the position of a probe relative to the twisting centre $\Delta z_{c}$ is known, for which the amount of probe offset resulting from only untwist can be calculated as follows (considering the upper part of Figure 5)

$$
\Delta P_{u}=\Delta z_{c}[\tan \emptyset-\tan (\varnothing-\psi)]
$$

If the untwist angle is small, it can be neglected and the axial-lean movement can be determined using the method described in section 3.2, and by using the total value of $\Delta P$ from any probe. In this case, $\Delta z_{c}$ is assumed as large as possible according to the blade dimensions, and the calculated $\Delta P_{u}$ is considered as the upper limit of error in the measured $\Delta P$ and can therefore be regarded as the uncertainty in the axial-lean movement estimation:

$\%$ uncertainty in axial-lean $=100 * \frac{\Delta P_{u}}{\Delta P}$ 


\subsection{Method guidelines}

The flow chart shown in Figure 6 summarizes the main steps of using the current method for the determination of blade tip steady movements.

\section{VALIDATION OF THE METHOD}

\subsection{FE model and BTT simulations}

The simulated data are generated by a BTT modal model simulator [23] that uses as input the modal data of a blisk FE model. The modal data are extracted from a one-off modal analysis of the blisk that is frozen at a reference angular position and fixed at its mounting hole. The simulator calculates the coordinates of the FE nodes at each time step by the superposition of three different motions: the rigid rotation of the undeformed blisk, the prescribed steady motions, and the vibration response, which is calculated by the integration of the modal differential equations of motion of the system. The blades are excited using FE nodal forces in the Cartesian coordinate system $(x, y, z)$. Different values and types of engine orders (synchronous and asynchronous) can be applied simultaneously. A travelling wave excitation can be applied by controlling the phase values of forces at all blades, and a certain mode shape can be excited by selecting the appropriate number of nodal diameters. The simulator uses the Simulink function Hitcrossing to find the blade arrival times at which the instantaneous coordinates of a given blade tip's FE nodes coincide with both the angular and axial positions of a probe. BTT displacement data are then calculated according to eq. (2).

The blisk shown in Figure 7 consists of a disk with 40 integrated blades. The disk has an inner radius of 25 mm, outer radius of $180 \mathrm{~mm}$, and $16 \mathrm{~mm}$ thickness. Each blade has a length of $90 \mathrm{~mm}$, thickness of $6 \mathrm{~mm}$, and chord line stagger angle (angle between chord line and the normal to the plane of rotation) of $60^{\circ}$. The material is aluminium of density $2750 \mathrm{~kg} / \mathrm{m}^{3}$ and Young's modulus $72 \mathrm{GPa}$. The geometry of the model has been built up using SolidWorks 2014, and then exported to ANSYS 15.0 to set up the different properties and boundary conditions of the FE model and run the required analysis. The mesh was created using the 10-noded solid element SOLID187, and then highly refined at the tips of a number of blades in order to reduce the gaps between the FE nodes which may affect the accuracy of the simulator for the considered case of steady movements (ideally, the tip surface would have an infinite number of nodes). The total number of degrees-of-freedom (DOFs) of the model is 1791261 .

For the purpose of generating the modal data required by the simulator, the blisk was assumed completely fixed at the surface of the inside diameter. Table 1 includes the first five natural frequencies of the non-rotating blisk, and Figure 8 shows their corresponding mode shapes. The modal data, as well as the coordinates of the nodes at the blade tips, were exported to the simulator. The other 
inputs to the simulator were defined as follows: the travelling wave excitation had an amplitude of $1 \mathrm{~N}$ and single EO; the rotational speed swept from 0 to $250 \mathrm{rev} / \mathrm{s}$; two probes with beams of diameter $0.2 \mathrm{~mm}$ were used at angular positions of $10^{\circ}$ and $60^{\circ}$ measured from the positive $x$-axis (Figure 7) and spaced $6 \mathrm{~mm}$ apart along the $z$-axis. Each simulation had a duration of $1.5 \mathrm{~s}$ each, but the prescribed movements started at $0.5 \mathrm{~s}$ and increased linearly over the rest of simulation time. The vibration response was negligible due to the low amplitude of the excitation and the highest speed being $90 \mathrm{~Hz}$ below the first blisk resonance. Thus, the detected blade displacements (given by eq. (2)) were almost purely the result of the applied steady movements.

Four tests were implemented with different total prescribed movements as listed in Table 2. The simulator generated the BTT displacements of a given blade as seen by all the probes within the time range of the rotational speed sweep, and then the data were processed for the steady offset and the steady movement as per method described in section 3. Finally, the results were compared to the prescribed values. Test 1 was used to validate the method described in section 3.2 , while tests $2-4$ were used to validate the untwist method in section 3.3 .

\subsection{Validation results}

Test 1 (Axial-Lean) Axial-lean movements require the use of two extra probes as described in section 3.2. The function of these probes is to determine the direction of movement once the mean position of the blade tip is detected by one of them. These additional probes ("outer probes") were placed at an angular position of $30^{\circ}$ relative to the $x$-axis (Figure 7) and were respectively positioned axially by $1 \mathrm{~mm}$ to the right, and $1 \mathrm{~mm}$ to the left, of the blade tip edges.

The variations of the output BTT displacement of a given blade tip detected by the two probes over an increasing number of revolutions are shown in Figure 9. It is clear that the two variations have the same rate of change, thus proving the condition in eq. (7a). According to the values of both axial and lean movement shown in Table 2, the angle $\emptyset_{S}$ should be $15^{\circ}$ and the combined amount of movement in this direction is $1.93 \mathrm{~mm}$. By monitoring the output of the outer two probes, it is found that one of them started to detect all the blades between revolutions numbers 89 and 90 , so the values of $\Delta P$ are extracted after that time. $\emptyset_{S}$ was then determined by applying eq. (10), yielding an estimate of $14.82^{\circ}$. Finally, the total amount of movement by the end of simulation was calculated as $1.92 \mathrm{~mm}$ which was $0.5 \%$ less than the prescribed movement. This error is expected because of the small error in direction and also because the prescribed movements last to the end of simulation time which is slightly greater than the time of the last signal from the probes. 
Tests 2, 3 (Axial-Untwist, Lean-Untwist) The BTT displacement results from tests 2 and 3 at the two probes are shown in Figure 10. The data rates of change from the two probes are different as expected based on the condition in eq. (7b). The untwist angle values relative to the number of revolutions are calculated using the method in section 3.3 as shown in Figure 11. The final values of calculated untwist angles in tests 2 and 3 are $5.03^{\circ}$ and $5.01^{\circ}$ respectively, which are $0.6 \%$ and $0.2 \%$ more than the prescribed values (Table 2).

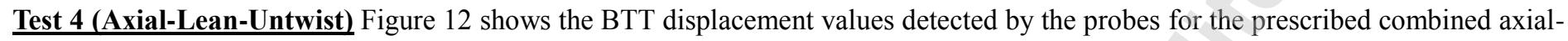
lean-untwist movement. The changes in probe data along the number of revolutions are different, as expected from condition in eq. (7b). The untwist angles are calculated according to the method in section 3.3, and are shown in Figure 13. The final angle value is $5.04^{\circ}(0.8 \%$ error). Knowing the value of untwist angle, it is possible to use eq. (14) to check the uncertainty in the axial-lean movement calculation if untwist is ignored. The value of $\Delta P_{u}$ to use in eq. (14) is calculated using eq. (13) with $\Delta z_{c}$ (the position of probe 1 relative to the twisting centre) assumed to be $\left(z_{1}-z_{\min }\right)$ (see Figure 4), as explained at the end of section 3.3. A plot of percentage uncertainty for different values of angles and $\Delta P$ is shown in Figure 14. It can be seen from this figure that the uncertainty in the $\emptyset_{S}$ and $\Delta P_{1}$ values (calculated by ignoring the untwist) at the level of actual untwist in the last revolution is about $80 \%$. According to Figure 14, the proposed method for calculating axial-lean movement is considered reasonably reliable for untwist angles of up to $2^{\circ}$.

\section{CONCLUSIONS}

A method for the determination of simultaneous blade steady movements (i.e. composed of a combination of axial shift, blade lean, and blade untwist) has been developed. The method depends on the BTT data captured by one or two probes according to the types of movement. Two extra probes are used at known axial distances from each end of the blade tip in case of combined axial and lean movements in order to determine the direction of movement. The changes of the average value of the BTT data at the probes were linked to the magnitude and direction of the movement via a number of geometrical relations. The method was validated using a modal model simulator of a blisk that generated BTT displacement data under conditions of prescribed steady movements. Tests were implemented for various types of combined movements (axial-lean, axial-untwist, lean-untwist, axial-lean-untwist), and the calculated movements compared with the prescribed values. The results prove the validity of the method for the determination of combined axial-lean movements in case of no or negligible untwist, and also the determination of untwist angles under all circumstances with high level of accuracy. As regards the determination of axial-lean under conditions of untwist, this could only be done if the untwist 
was ignored, but an expression for the resulting uncertainty in the calculation was established and illustrated. More developments are required in order to calculate the axial-lean movements in case of large untwist angles.

The tests in this paper were conducted with simulated data and the displacement data was entirely dominated by the steady movement (due to negligible vibration response). However, the work of this paper was developed from the authors' previous work [29], wherein individual axial/lean/untwist steady movements were successfully quantified using experimental BTT data, and also under resonance conditions.

\section{ACKNOWLEDGMENTS}

We would like to thank the Egyptian Ministry of Higher Education, and the British Council in Cairo for funding the project.

\section{REFERENCES}

[1] Russhard, P., 2015, "The Rise and Fall of the Rotor Blade Strain Gauge," J.K. Sinha, ed., Springer International Publishing, Cham, pp. 27-37.

[2] Prochazka, P., and Vanek, F., 2013, "New Methods of Non-Contact Sensing of Blade Vibrations and Deflections in Turbomachinery," 2013 IEEE International Instrumentation and Measurement Technology Conference (I2MTC), IEEE, pp. 339-344.

[3] García, I., Beloki, J., Zubia, J., Aldabaldetreku, G., Illarramendi, M., and Jiménez, F., 2013, “An Optical Fiber Bundle Sensor for Tip Clearance and Tip Timing Measurements in a Turbine Rig,” Sensors, 13(6), pp. 7385-7398.

[4] Watkins, W., Robinson, W., and Chi, R., 1985, "Noncontact Engine Blade Vibration Measurements and Analysis," 21st Joint Propulsion Conference, American Institute of Aeronautics and Astronautics, Monterey, California.

[5] Kubín, Z., Mísek, T., Hlous, J., Dadaková, T., Kellner, J., and Bachorec, T., 2018, "Calibration of Blade Tip-Timing Sensor for Shrouded 40" Last Stage Blade," Mechanical Systems and Signal Processing, 108, pp. 88-98.

[6] Zielinski, M., and Ziller, G., 2000, "Noncontact Vibration Measurements on Compressor Rotor Blades," Measurement Science and Technology, 11(7), p. 847.

[7] Chana, K. S., Sridhar, V., and Singh, D., 2016, "The Use of Eddy Current Sensors for the Measurement of Rotor Blade Tip Timing: Development of a New Method Based on Integration," Proceedings of the ASME Turbo Expo 2016: Volume 6: Ceramics; Controls, Diagnostics and Instrumentation; Education; Manufacturing Materials and Metallurgy. Seoul, South Korea. June 13-17, 2016. V006T05A019., pp. 179-189.

[8] Zablotskii, I., Korostelev, Y., and Sviblov, L., 1974, "Contactless Measuring of Vibrations in the Rotor Blades of Turbines," Foreign technology Division, Air Force Systems Command, U.S. Air Force, FTD-HT-23-673-74.

[9] Nieberding, W., Pollack, J., 1977, “Optical Detection of Blade Flutter,” NASA Lewis Research Center, NASA TM X-73573.

[10] Kurkov, A., and Dicus, J., 1978, "Synthesis of Blade Flutter Vibratory Patterns Using Stationary Transducers," Volume 1B: General, ASME. 
[11] Heath, S., 1996, "A Study of Tip-Timing Techniques for the Determination of Bladed Disk Vibration Characteristics," Ph.D. dissertation, The University of London, London, United Kingdom.

[12] Heath, S., and Imregun, M., 1996, "An Improved Single-Parameter Tip-Timing Method For Turbomachinery Blade Vibration Measurement Using Optical Laser Probes,” International Journal of Mechanical Sciences, 38(10), pp. 1047-1058.

[13] Heath, S., 2000, “A New Technique For Identifying Synchronous Resonances Using Tip-Timing,” Journal of Engineering for Gas Turbines and Power-Transactions of the ASME, 122(2), pp. 219-225.

[14] Carrington, I., Wright, J., Cooper, J., and Dimitriadis, G., 2001, “A Comparison of Blade Tip Timing Data Analysis Methods," Proceedings of the Institution of Mechanical Engineers, Part G: Journal of Aerospace Engineering, 215(5), pp. 301-312.

[15] Gallego-Garrido, J., Dimitriadis, G., and Wright, J., 2007, “A Class of Methods for the Analysis of Blade Tip Timing Data from Bladed Assemblies Undergoing Simultaneous Resonances-Part I: Theoretical Development," International Journal of Rotating Machinery, pp. 1-11.

[16] Gallego-Garrido, J., Dimitriadis, G., Carrington, I., and Wright, J., 2007, "A Class of Methods for the Analysis of Blade Tip Timing Data from Bladed Assemblies Undergoing Simultaneous Resonances-Part II: Experimental Validation," International Journal of Rotating Machinery, pp. 1-10.

[17] Russhard, P., 2010, “Development of a Blade Tip Timing Based Engine Health Monitoring System,” Eng.D. dissertation, University of Manchester, Manchester, United Kingdom.

[18] Rigosi, G., Battiato, G., and Berruti, T., 2016, "Synchronous Vibration Parameters Identification by Tip Timing Measurements," Mechanics Research Communications, 79, pp. 7-14.

[19] Lin, J., Hu, Z., Chen, Z. S., Yang, Y. M., and Xu, H. L., 2016, "Sparse Reconstruction of Blade Tip-Timing Signals for MultiMode Blade Vibration Monitoring," Mechanical Systems and Signal Processing, 81, pp. 250-258.

[20] Joung, K., Kang, S., Paeng, K., Park, N., Choi, H., You, Y., and Flotow, A., 2006, "Analysis of Vibration of The Turbine Blades Using Non-Intrusive Stress Measurement System,” ASME 2006 Power Conference, pp. 1-7.

[21] Lawson, C., and Ivey, P., 2003, “Compressor Blade Tip Timing Using Capacitance Tip Clearance Probes,” Proceedings of ASME Turbo Expo 2003, pp. 1-8.

[22] Russhard, P., 2016, “Blade Tip Timing (BTT) Uncertainties,” AIP Conference Proceedings, 1740.

[23] Mohamed, M., Bonello, P., and Russhard, P., 2018, “The Determination of Steady-State Movements Using Blade Tip Timing Data," ASME. Turbo Expo: Power for Land, Sea, and Air, Volume 7C: Structures and Dynamics, ASME, Oslo, Norway, p. V07CT35A010.

[24] Russhard, P., 2012, “Blade Tip Timing - Frequently Asked Questions,” (June).

[25] Twerdochlib, M., Osborne, R., and Rozelle, P., 1990, "Methods for Determining The Untwist of Turbine Blades," United States Patent, US07385753.

[26] Clifford Hatcher, J., and Brindisi, J., 2015, "Method of Determining the Location of Tip Timing Sensors during Operation," United states Patent, US20150199805 A1.

[27] Kominsky, D., 2011, "Rotating Stall Detection Using Optical Measurement of Blade Untwist," United States Patent, US $8,854,626$ B2, pp. 12-15. 
[28] Jousselin, O., 2013, "Development of Blade Tip Timing Techniques in Turbo Machinery," Ph.D. dissertation, The University of Manchester, Manchester, United Kingdom.

[29] Mohamed, M., Bonello, P., and Russhard, P., 2019, "A Novel Method for the Determination of the Change in Blade Tip Timing Probe Sensing Position due to Steady Movements,” Mechanical Systems and Signal Processing, 126, pp. 686-710.

[30] Diamond, D. H., and Stephan Heyns, P., 2018, “A Novel Method for the Design of Proximity Sensor Configuration for Rotor Blade Tip Timing," Journal of Vibration and Acoustics, 140(6), pp. 1-8.

[31] Carrington, I. B., 2002, "Development of Blade Tip Timing Data Analysis Techniques," Ph.D. dissertation, University of Manchester, Manchester, United Kingdom.

\section{LIST OF TABLES}

Table 1: Natural frequencies of the blisk.

Table 2: Prescribed movements for validation tests.

\section{LIST OF FIGURES}

Figure 1: Types of blade tip steady movements. (a) Axial shift, (b) Blade lean, (c) Blade untwist, (d) Simultaneous movements.

Figure 2: Typical arrangement for measuring $t_{\mathrm{exp}, j}$ using once-per-revolution (OPR) probe [29].

Figure 3: Probe offset (a) Constant offset, (b) Variable offset.

Figure 4: Geometry of combined axial-Lean blade tip movement.

Figure 5: Axial-Lean-untwist blade tip movement.

Figure 6: Method guidelines.

Figure 7: Blisk FE model.

Figure 8: Blisk mode shapes. (a) First mode, (b) Second mode, (c) Third mode, (d) Fourth mode, (e) Fifth mode (please refer to online version for more clarity).

Figure 9: BTT displacements (test 1).

Figure 10: BTT displacement. (a) test 2, (b) test 3.

Figure 11: Untwist angle. (a) test 2, (b) test 3.

Figure 12: BTT displacement (test 4).

Figure 13: untwist angle (test 4).

Figure 14: Colour plot of probe offset percentage due to untwist (please refer to online version for more clarity). 
Table 1: Natural frequencies of the blisk.

\begin{tabular}{|c|c|c|c|c|c|}
\hline Mode No. & 1 & 2 & 3 & 4 & 5 \\
\hline Type & bending & bending & axial & bending & bending \\
\hline Frequency (Hz) & 340.8 & 340.8 & 361.5 & 392.9 & 393.0 \\
\hline Nodal diameters & 1 & 1 & 0 & 2 & 2 \\
\hline
\end{tabular}


Table 2: Prescribed movements for validation tests.

\begin{tabular}{|c|c|c|c|}
\hline Test & Axial shift (mm) & Lean (mm) & Untwist (degrees) \\
\hline 1 & 1 & 1 & 0 \\
\hline 2 & 1 & 0 & 5 \\
\hline 3 & 0 & 1 & 5 \\
\hline 4 & 1 & 1 & 5 \\
\hline
\end{tabular}




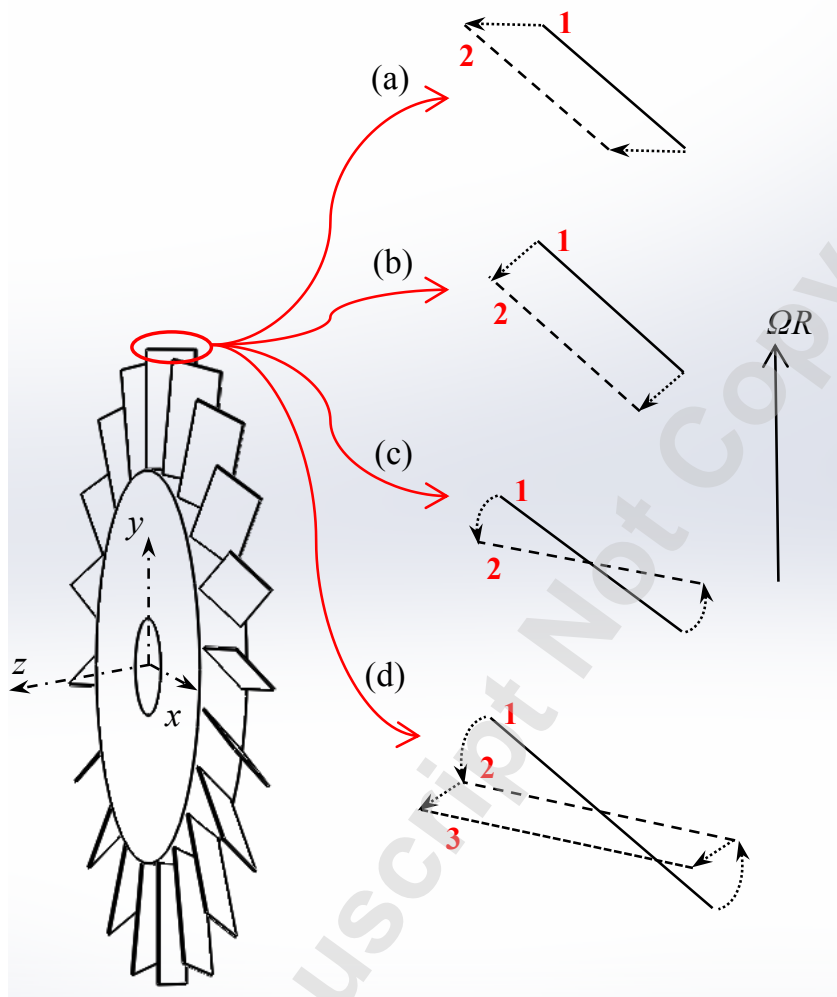

Figure 1: Types of blade tip steady movements. (a) Axial shift, (b) Blade lean, (c) Blade untwist, (d) Simultaneous movements. 


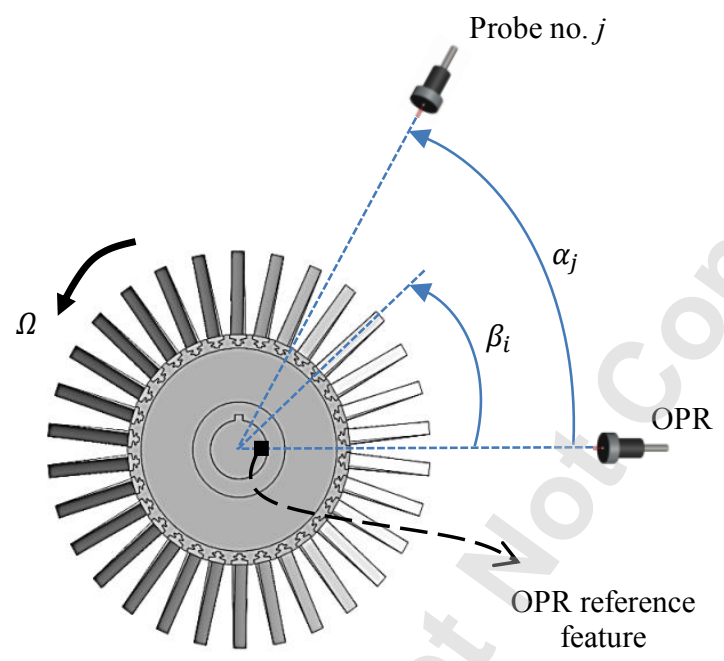

Figure 2: Typical arrangement for measuring $t_{\mathrm{exp}, j}$ using once-perrevolution (OPR) probe [29]. 
a)
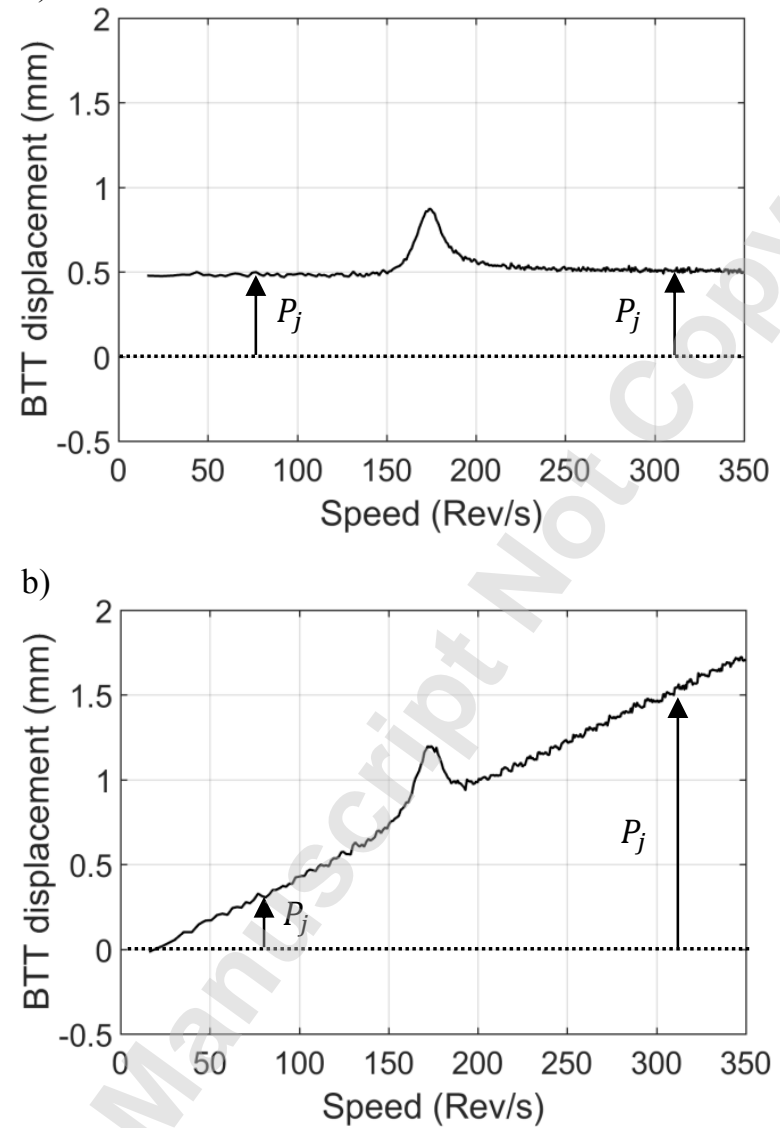

Figure 3: Probe offset (a) Constant offset, (b) Variable offset. 

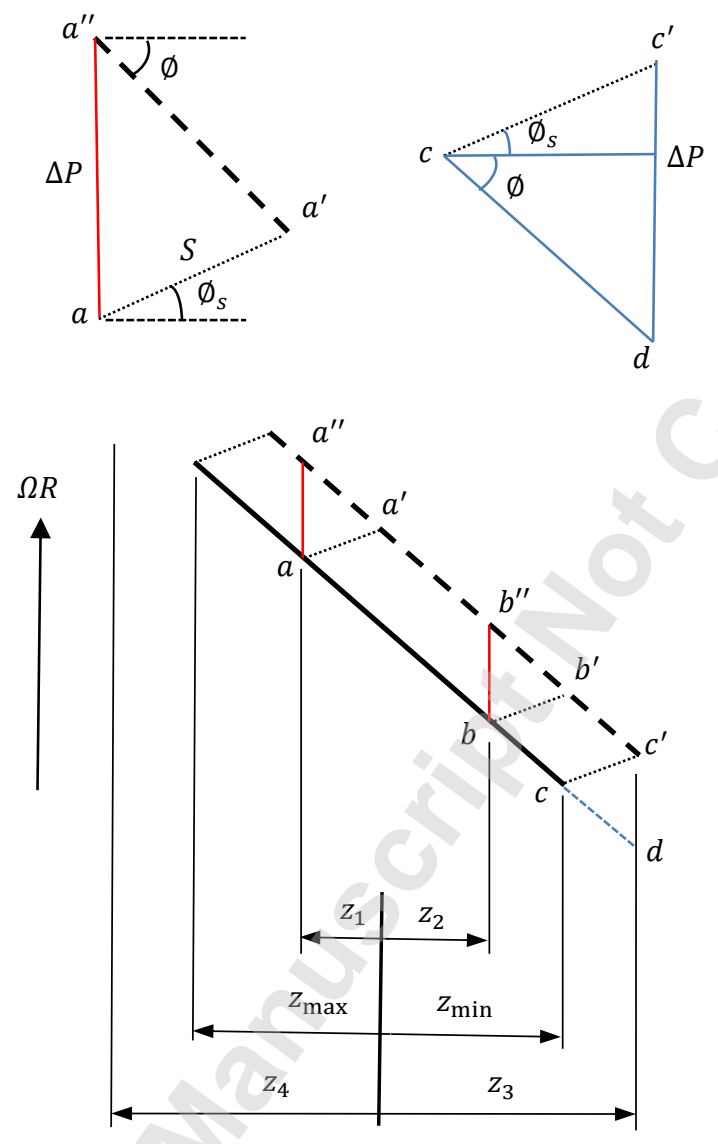

Figure 4: geometry of combined axial-Lean blade tip movement. 

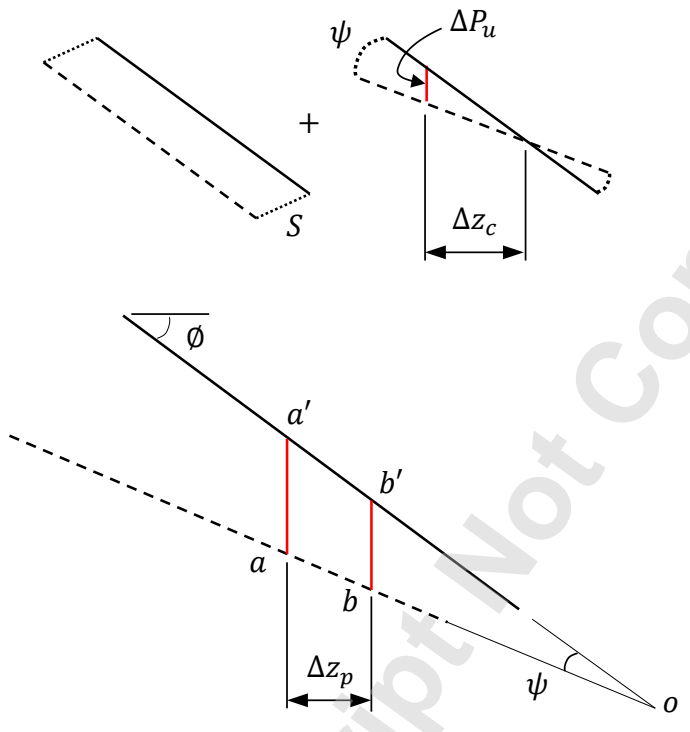

Figure 5: Axial-Lean-untwist blade tip movement. 


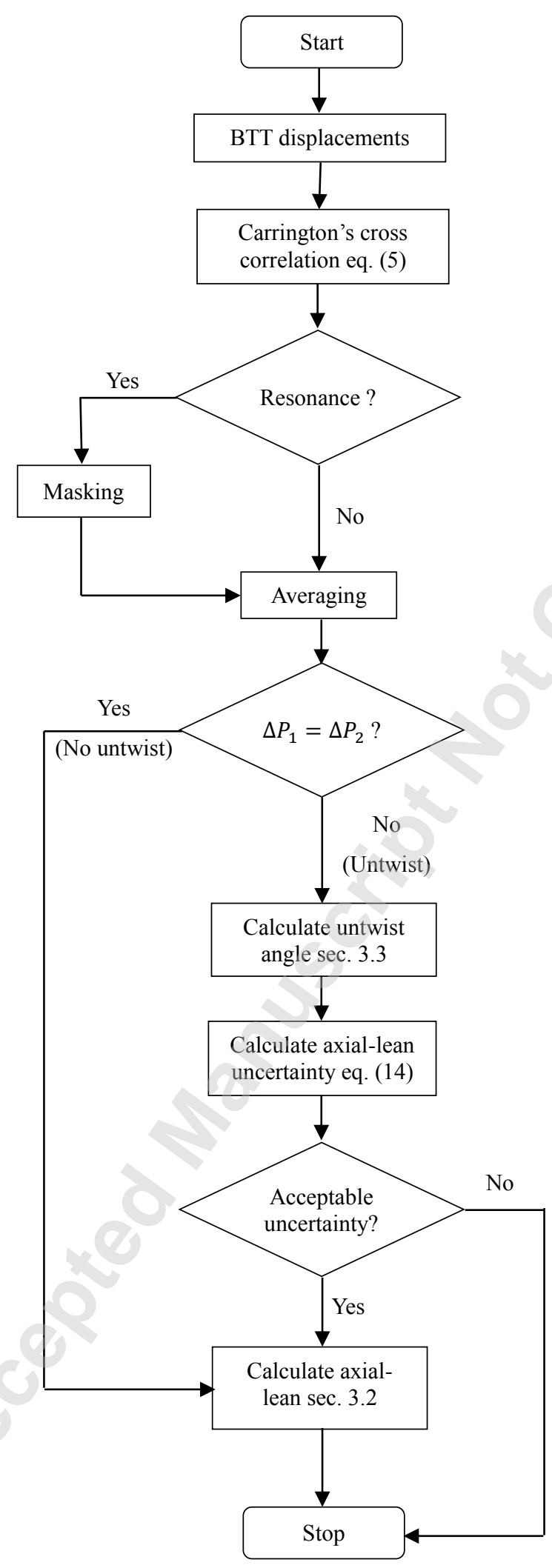

Figure 6: Method guidelines. 


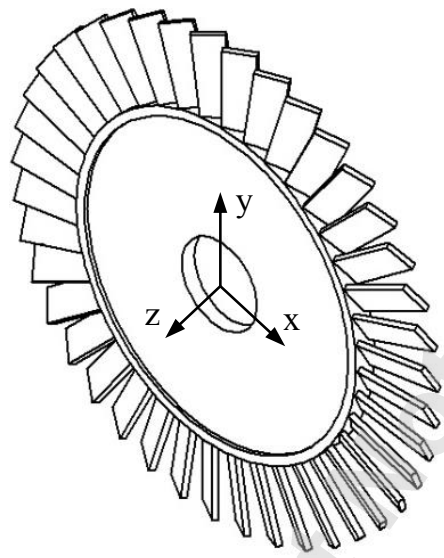

Figure 7: Blisk FE model. 
a)

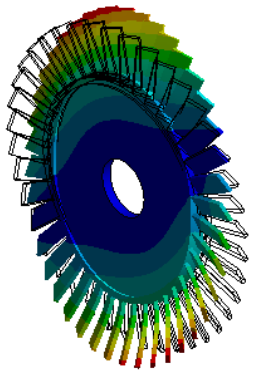

b)

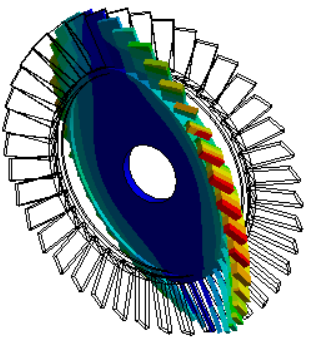

c)

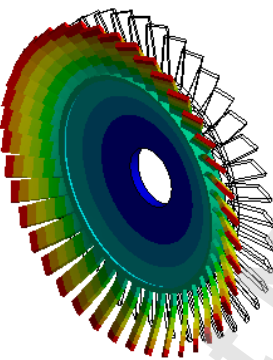

d)

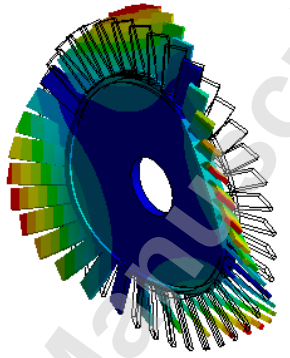

e)

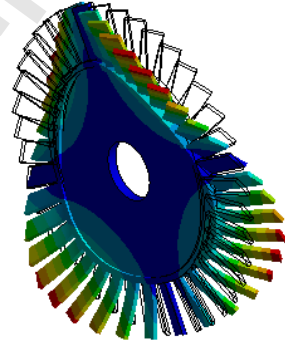

Figure 8: Blisk mode shapes. (a) First mode, (b) Second mode, (c) Third mode, (d) Fourth mode, (e) Fifth mode (please refer to online version for more clarity). 


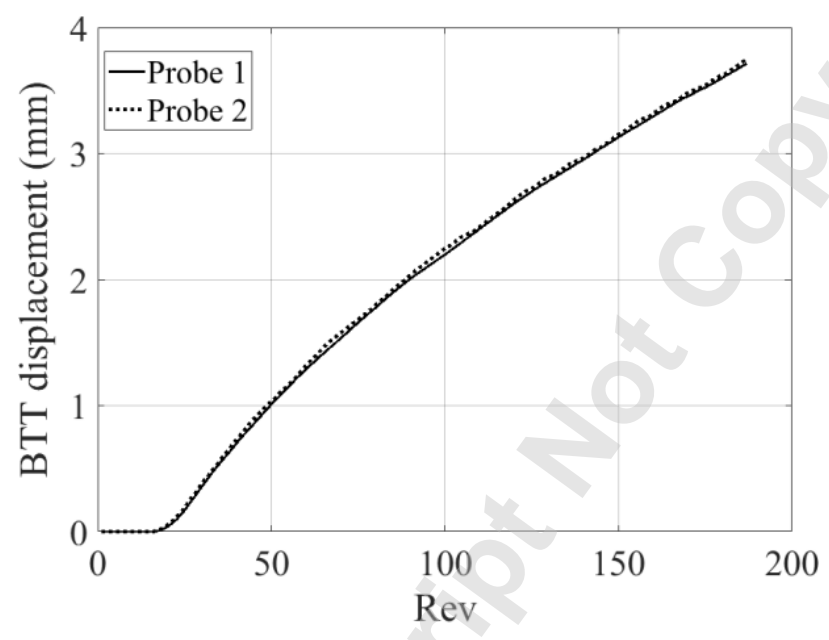

Figure 9: BTT displacements (test 1). 

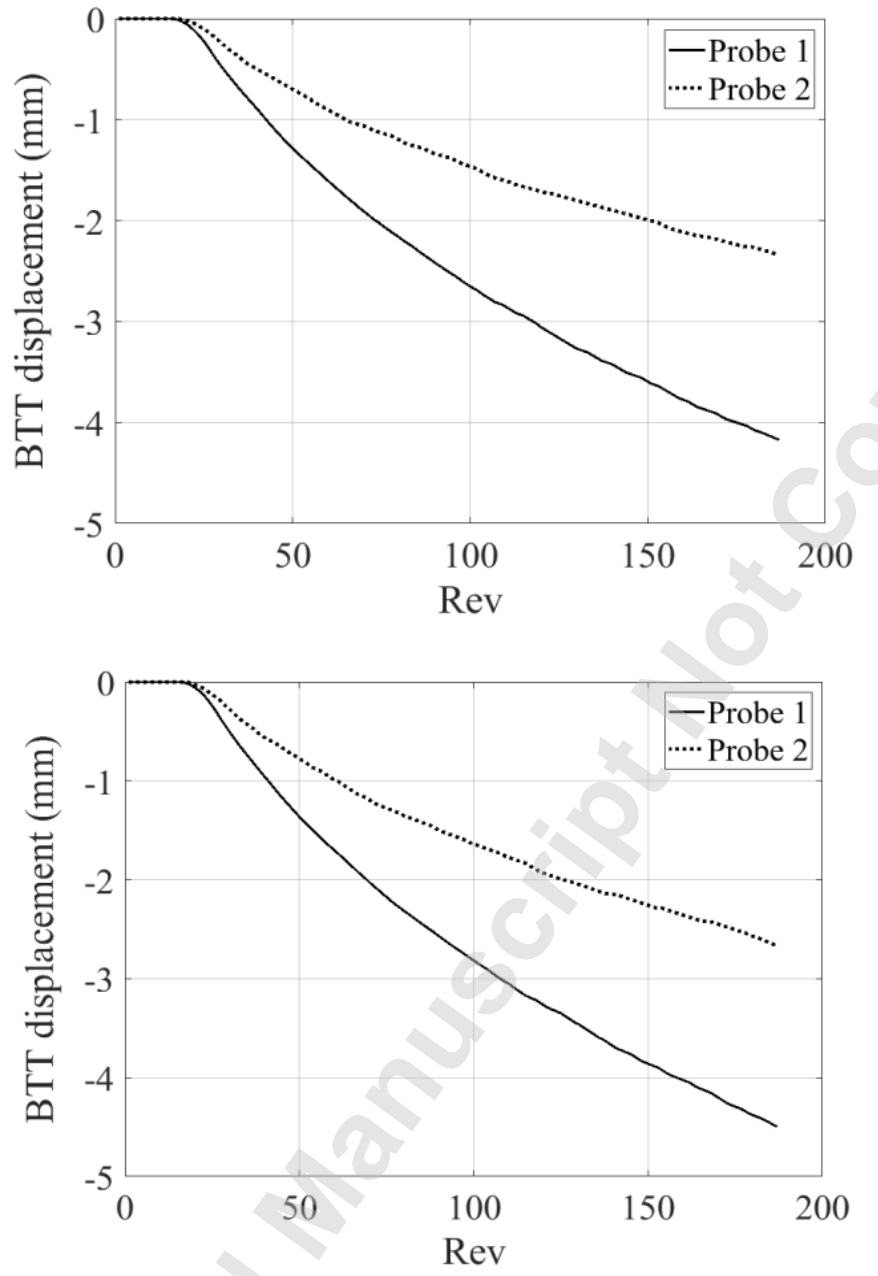

Figure 10: BTT displacement. (a) test 2, (b) test 3. 

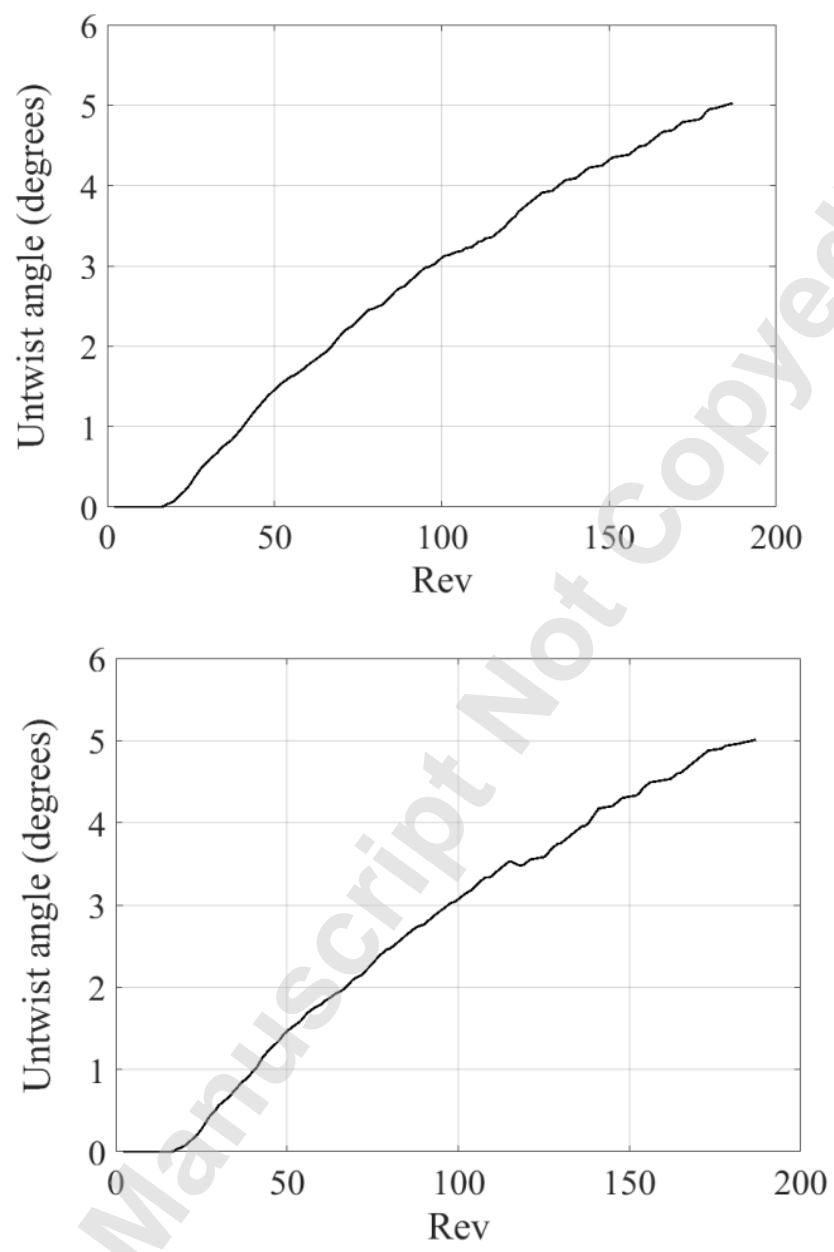

Figure 11: Untwist angle. (a) test 2, (b) test 3. 


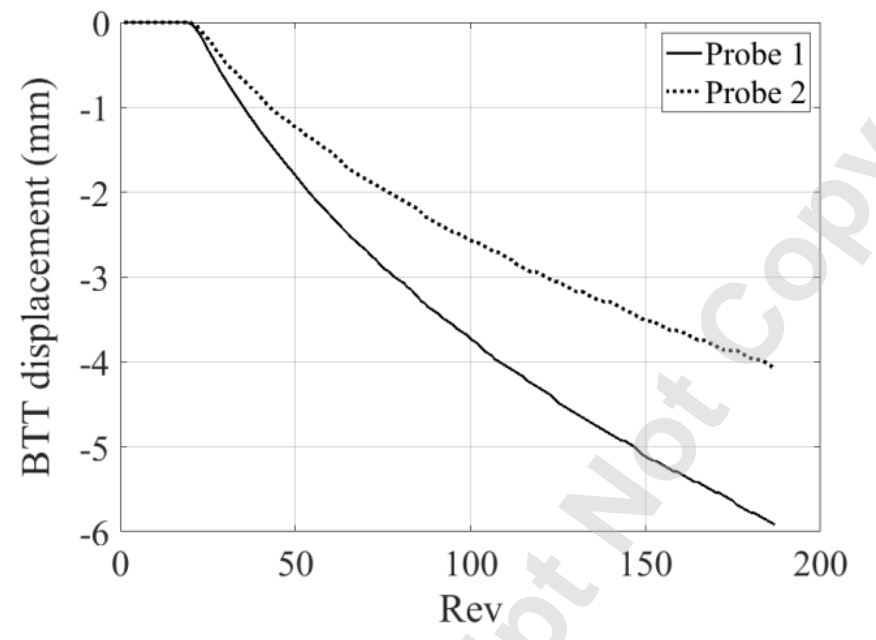

Figure 12: BTT displacement (test 4). 


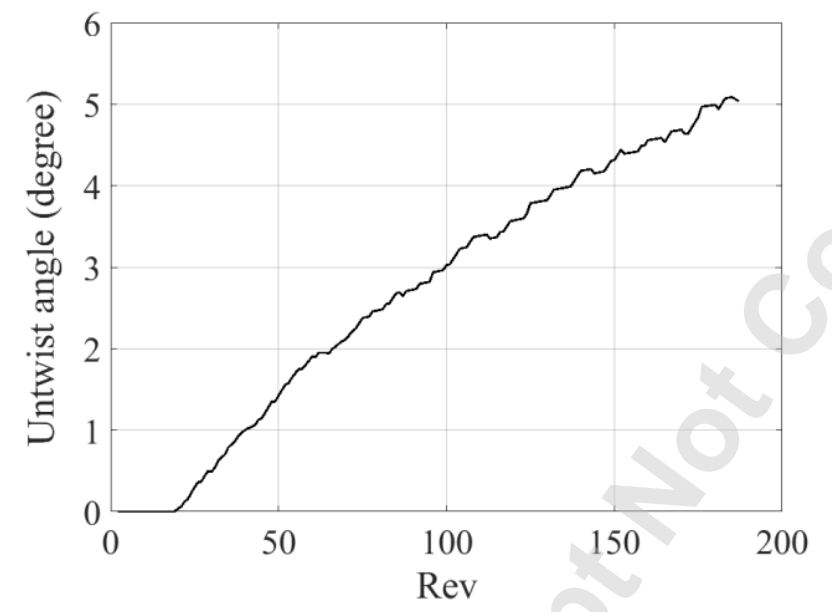

Figure 13: untwist angle (test 4). 


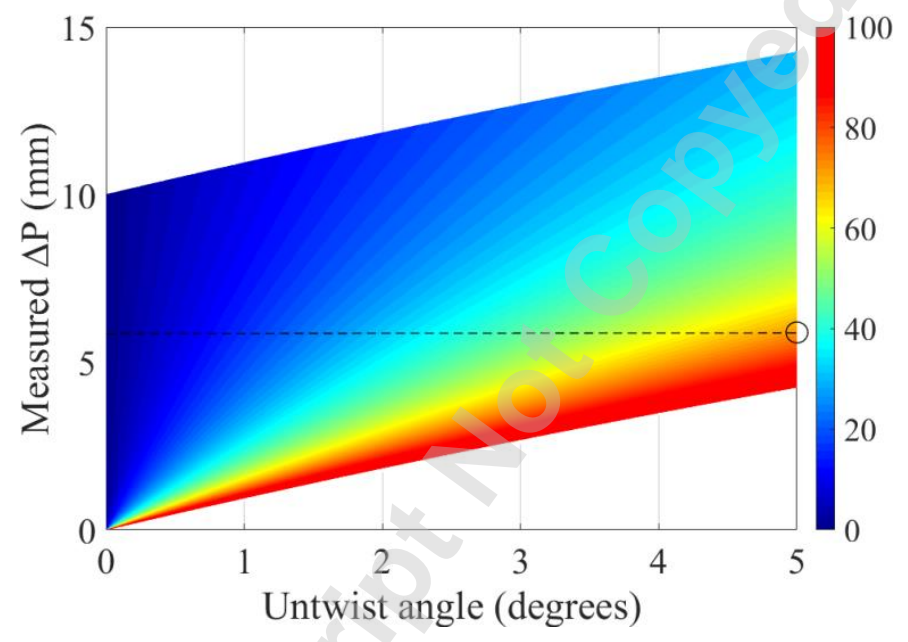

Figure 14: Colour plot of probe offset percentage due to untwist (please refer to online version for more clarity). 